\title{
Öffentliche Mobilität und eine neue Mobilitätskultur - Grundlagen, Entwicklungen und Wege zur kulturellen Verkehrswende
}

\section{Maximilian Hoor}

\section{Einleitung}

Der vorliegende Beitrag setzt sich aus einer kulturtheoretischen Perspektive mit neuen Mobilitätskulturen und ihrer Bedeutung für die Öffentliche Mobilität auseinander. Wenn es das verkehrsplanerische und -politische Ziel ist, jede*r Bürger*in die gesellschaftliche Teilhabe zu gewähren, aber den dadurch verursachten Verkehr in seinen negativen Auswirkungen zu begrenzen (siehe die Einleitung von Schwedes in diesem Band), dann werden vielfältige und mit Konflikten verbundene Umgestaltungs-, Umverteilungs- und Aushandlungsprozesse geführt werden müssen, die sowohl auf materieller wie auf symbolischer Ebene wirken: Es braucht eine neue Mobilitätskultur.

\section{Bedeutung kultureller Aspekte von Mobilität und Verkehr}

In politischen, medialen, planerischen und wissenschaftlichen Diskursen wird der Begriff ,Mobilitätskultur' häufig benutzt, insbesondere wenn es um den Transformationsprozess hin zu einer nachhaltigen Mobilität geht. Grundlegend geht es hier um die Einsicht, dass eingefahrene Lebensweisen, Routinen und Überzeugungen auf individueller wie gesellschaftlicher Ebene verstanden und aufgebrochen werden müssen, um eine nachhaltige bzw. postfossile Mobilität erreichen zu können. So lässt sich beispielsweise die Hartnäckigkeit und Beständigkeit der Automobilisierung durch politische, soziale, technische, ökonomische, aber auch durch kulturelle Beharrungskräfte und Pfadabhängigkeiten

M. Hoor $(\bowtie)$

Technische Universität Berlin, Berlin, Deutschland

E-Mail: m.hoor@tu-berlin.de 
erklären: Das Auto galt (und gilt) für viele als Symbol für Freiheit, Moderne und Individualität, das aus den Köpfen der Menschen nicht mehr rauszukriegen und durch eine starke Emotionalisierung und Konditionierung verhaftet ist das Auto hat also eine starke kulturelle Bedeutung für die alltäglichen Lebensweisen von Menschen und das Funktionieren unserer Gesellschaft, welche eine Eigenmächtigkeit besitzt (Canzler und Radtke 2019; Grieger 2019). So lässt sich erklären, warum die meisten verkehrspolitischen Restriktionen, wie z. B. Parkraumbewirtschaftung oder Tempolimits, als kollektive Freiheitsberaubung gewertet werden, während automobile Lebensstile und Stadtstrukturen gleichzeitig naturalisiert und verteidigt werden (Reents 2019; Manderscheid 2012). Dabei benötigt eine nachhaltige Verkehrsentwicklung eine umfassende Verkehrswende, die zwangsläufig mit konsequenten Maßnahmen einhergeht (Rudolph et al. 2018).

Eine Betrachtung und Vermittlung dieses Dilemmas ist insbesondere jetzt wichtig, da einerseits der Ruf nach einer Verkehrswende und der Transformation $\mathrm{zu}$ nachhaltigeren Mobilitätskulturen in aller Munde ist, und andererseits neue Entwicklungen insbesondere in der städtischen Alltagsmobilität stattfinden, die nicht eindeutig zu interpretieren sind: Sind junge Menschen weniger auto-affin? Welche Mobilitätsangebote sind für sie relevant? Was können Digitalisierung und neue Mobilitätsdienstleistungen wirklich leisten? Wie lässt sich der aktuelle Bedeutungsgewinn des Fahrrads erklären?

Dabei ist ersichtlich, dass sich ein Großteil des Diskurses an technischen Innovationen bemisst und die ,einfachen', auf der Hand liegenden verkehrsplanerischen Strategien und Maßnahmen sowie soziale Innovationen vernachlässigt werden: Die Hoffnungen auf das autonome Fahren sowie neue Informations- und Kommunikationstechnologien oder neuartige Verkehrsmittel (z. B. E-Tretroller) scheinen in keinem angemessenen Verhältnis zu den bisherigen Erfahrungen und ernsthaften Prognosen zu stehen (Schwedes 2020; Litman 2020). Dementgegen verzeichnet aber insbesondere der Radverkehr, welcher als Teil des Umweltverbundes pars pro toto für eine nachhaltige Verkehrsentwicklung steht, in den Städten enorme Zuwachsraten und geht mit einer Vielzahl alltäglicher Praktiken, Bedarfe und verkehrspolitischer Forderungen einher, welche trotz aller radverkehrspolitischen Erfolge immer noch nicht ausreichend implementiert und beachtet werden.

\section{Zielsetzung und Ablauf}

Der vorliegenden Artikel ist einen Beitrag zur Eruierung einer neuen Mobilitätskultur. Dafür werde ich das theoretische Verständnis von Mobilitätskulturen herleiten (siehe Kap. 2, basierend auf Hoor 2020a). In einem weiteren Schritt werde 
ich konzeptionelle Grundlagen beschreiben (Kap. 3) und daraus ableiten, welche aktuellen Entwicklungstrends für verkehrsplanerische und -politische Zwecke genutzt werden könnten bzw. noch bedacht werden müssten (Kap. 4).

Dabei möchte ich festhalten, dass meine eigene Perspektive, die eines überzeugten Fahrradfahrers ist und sich auch meine eigene Forschung, neben dem Thema Mobilitätskulturen, fast ausschließlich auf das städtische Radfahren beschränkt. Trotzdem, oder gerade deshalb, fallen mir im Vergleich bestimmte Themen auf, bei denen der Rest des Umweltverbundes vom Radverkehr einiges lernen kann - positiv wie negativ.

\section{Theorie - Mobilitätskulturen}

Ebenso häufig wie der Begriff, Mobilitätskultur(en)' mittlerweile genutzt wird, so definitorisch unklar und arbiträr ist seine Bedeutung: Manche verstehen darunter eine veränderte Verkehrsinfrastruktur zur Förderung aktiver Mobilität und öffentlichen Verkehr; andere ein verändertes Mobilitätshandeln bzw. -verhalten seitens der Nutzenden; wiederum andere eine neue Planungskultur, z. B. auf Seiten kommunaler Verwaltungen. Diese Problematik ist keineswegs neu, so wurde der Begriff bereits 2013 als Modebegriff beschrieben der oft ,nur als Schlagwort benutzt [wird] ohne ihn näher zu erklären oder zu definieren“ (Ahrend et al. 2013, S. 42). Dabei wird der Begriff medial und politisch insbesondere präskriptiv verwendet, z. B. wenn eine neue Mobilitätskultur gefordert wird. Die akademischen Begriffsverwendungen sind vor allem deskriptiv, analytisch und vergleichend (Götz et al. 2016; Institute for Mobilities Research 2013; Klinger et al. 2013; Deffner et al. 2006).

\section{$1.1 \quad$ Begriffsklärung}

Ein Teil der begrifflichen Unklarheit von Mobilitätskulturen ist dem vorgelagerten Begriff Mobilität zu verdanken, welcher ebenfalls häufig unpräzise, uneinheitlich oder auch gerne analog zum Begriff Verkehr genutzt wird (Schwedes et al. 2018; sowie den Beitrag von Rammert in diesem Band). Hinzu kommt der Begriff Kultur, welcher nach dem britischen Kulturtheoretiker Raymond Williams (1976) eines der kompliziertesten Worte unserer Sprache darstellt. Ganz allgemein kann Kultur als Verbindung zwischen lebensweltlichen Praktiken und Dingen sowie ihrer symbolischen Bedeutung verstanden werden. Vereinfacht ausgedrückt beinhaltet der Kulturbegriff auf der einen Seite die 
materiellen Dinge und das, was Leute tun; auf der anderen Seite die unterschiedlichen Bedeutungen, die diese Dinge und das Tun für bestimmte Individuen und Personengruppen haben kann (Müller-Funk 2010; Eagleton 2009; Williams 1961, 1981).

\section{Kulturtheoretische Grundlagen}

Die Auslegung des Begriffs Kultur und daran anschließende Kulturtheorien sind als äußerst heterogen und vielfältig zu verstehen (Nünning und Nünning 2008). Dabei verfolge ich die nicht-elitäre Kulturdefinitionen von Raymond Williams (1961, 1981) und den Cultural Studies, die Kultur einerseits als Gesamtheit aller Lebensweisen und anderseits als Bedeutungssystem verstehen. Kultur ist somit als das Medium zu verstehen, das der materiellen Welt und den banalen Alltagspraktiken einen symbolischen Wert beimisst und sie mit Sinn ausstattet. Dabei bezieht sich Kultur immer auf die Aspekte des Materiellen und des Geistigen: Strukturelle Gegebenheiten und Prozesse wie Infrastrukturen, Gesetze, politische und ökonomische Verhältnisse; aber auch gelebte Praktiken sind Grundlage für deren kulturelle Repräsentationen in Narrativen, Mythen, Diskursen, Praktiken, Ideologien und Weltbildern. Die Beziehung zwischen diesen Sphären muss dabei als wechselseitig verstanden werden: So produzieren materielle Strukturen und Prozesse bestimmte Repräsentationen, werden jedoch auch durch diese selbst geprägt und (re)produziert. Folgt man dem hier hergeleiteten Kulturverständnis, so müssen Mobilitätskulturen sowohl als Abbilder und ebenso als Produzenten von Infrastruktur, Verkehr und Mobilität gesehen werden (Divall und Revill 2005; Mitchell 2000).

\section{Bestehende Begriffsverwendungen in der Mobilitätsforschung}

Bei der begrifflichen Erschließung der Mobilitätskultur wählte das Team um Jutta Deffner (2006) eine Perspektive, um „das Mobilitätsgeschehen sozialräumlicher Einheiten $[\ldots]$ vergleichend zu analysieren und als komplexe Interdependenz infrastruktureller, baulicher, diskursiver, sozialer, soziokultureller und handlungsbezogener Faktoren zu beschreiben“ (Götz et al. 2016, S. 782 f.). Nach ihrer Definition ist eine Mobilitätskultur „die Ganzheit der auf Beweglichkeit bezogenen materiell und symbolisch wirksamen Praxisformen. Sie schließt die Infrastruktur- und Raumgestaltung ebenso ein wie Leitbilder und verkehrspolitische Diskurse, das Verhalten der Verkehrsteilnehmer und die dahinterstehenden Mobilitäts- und Lebensstilorientierungen. Sie bezeichnet das prozessuale Ineinanderwirken von Mobilitätsakteuren, Infrastrukturen und Techniken als sozio-technisches System“ (Deffner et al. 2006, S. 16). 
Diesen vergleichenden Charakter nutzten andere Wissenschaftler*innen in anschließenden Studien unter anderem dazu, Mobilitätskulturen unterschiedlicher Städte miteinander zu vergleichen (Klinger et al. 2013) oder die Mobilitätskultur weltweiter Megastädte zu untersuchen (Institute for Mobilities Research 2013). Dabei bringt der Fokus auf eher quantitative, komparative und homogenisierende Kulturanalysen Probleme mit sich: Trotz des Anspruchs, sozialräumliche Einheiten miteinander vergleichen zu wollen und ein nutzbares Verständnis für die Verkehrswissenschaft und -planung zu entwickeln, stellt sich die Frage, inwiefern und ob überhaupt Mobilitätskultur(en) adäquat operationalisiert, gemessen und verglichen werden können, ohne dabei die konfliktreichen und normativ aufgeladenen Aushandlungsprozesse zu verschweigen, durch die sie überhaupt geschaffen, verfestigt und verändert werden; oder auch die Pluralität, Heterogenität und Widersprüchlichkeiten der Bedeutungen $\mathrm{zu}$ vernachlässigen, die sich innerhalb sozialräumlicher Einheiten, in gesellschaftlichen Gruppen und zwischen Individuen ergeben können und die immer auch in Mobilitätskulturen eingeschrieben sind.

\section{Synthese}

Dabei ist zu beachten, dass es niemals nur eine Kultur gibt, sondern immer eine Vielzahl unterschiedlicher Kulturen existieren, die nebeneinanderstehen, sich überkreuzen, ein- und ausschließen und um Bedeutung ringen. Bereits hier wird ein politischer Impetus des Kulturbegriffs deutlich, der besonders relevant für eine zielorientierte, sozialwissenschaftlich argumentierende und akteurszentrierte Mobilitäts- und Verkehrsforschung ist. Somit zeigt die Wahl des Begriffs Mobilitätskultur (im Singular) bereits ein Kulturverständnis, welches die Gleichzeitigkeit unterschiedlicher Mobilitätskulturen innerhalb einer sozialräumlichen Einheit sprachlich ausschließt.

Aus diesen Gründen macht es theoretisch, empirisch, wie auch sprachlich wenig Sinn von Mobilitätskulturen im Singular zu sprechen und z. B. eine nachhaltige Mobilitätskultur einer bestimmten Stadt oder Region zu fordern. Was damit aber vielfach gemeint ist - und so auch in diesem Artikel -, ist, dass bestimmte, teilweise sehr heterogene Mobilitätskulturen, die nach einer bestimmten normativen Zielorientierung (z. B. sozialökologischer Nachhaltigkeit oder öffentlicher Mobilität) als wünschenswert anzusehen sind und deshalb unter einer Mobilitätskultur vereinheitlicht werden, ein zukünftiger Hegemonieanspruch zugesprochen wird: „Mobilitätskulturen bezeichnen somit die Gesamtheit aller, sich zum Teil widersprechenden, Alltagspraktiken, Objekte und Sinnzuschreibungen, die - eingebettet in materielle Strukturen und gesellschaftliche Dispositionen - individuelle Möglichkeitsräume für Ortsveränderungen (re) präsentieren und (re)produzieren“" (Hoor 2020a, S. 24). 


\subsection{Was sind Mobilitätskulturen? Über konfliktreiche Aushandlungsprozesse}

In der Tradition der angelsächsischen Cultural Studies muss Kultur immer als Feld verstanden werden, in dem ,politische und soziale Identitäten produziert und reproduziert werden“, die „Zwangsweise Ausschlüsse, sowie Verhältnisse von Dominanz und Unterordnung, die ihrerseits auf Widerstände treffen“, produzieren (Marchart 2008, S. 12). Kultur ist damit das Medium des Konflikts und Kampfs, in dem bestehende Machtstrukturen diskursiv und lebensweltlich normalisiert, verfestigt, angefochten und ausgehandelt werden.

\section{Der Kampf um Hegemonie}

Diesen Zusammenhang zwischen Kultur, Macht und Gesellschaft beschreibt Don Mitchell sinnbildlich, indem er Kultur als ,an incredibly powerful idea [that] is made real, as real as any other exercise of power" (Mitchell 2000, S. 76 f.) beschreibt, in der ästhetische und symbolische Repräsentationen in materielle Strukturen umgewandelt werden. In den angelsächsischen Cultural Studies wird diese konzeptuelle Verbindung von Machtstrukturen und kulturellen Repräsentationen mit Antonio Gramscis (1992) Verständnis von Staatsmacht und Hegemonie vollzogen (z. B. Hall 2000, 2004; Williams 1981): In Gramscis Verständnis wird Kultur als ,wesentliche[r] Austragungsort politischer Kämpfe um Hegemonie konzipiert" (Marchart 2008, S. 76). Hegemonie ist dabei, verkürzt dargestellt, die weichere Seite gesellschaftlicher Machtausübung, die - neben klassischen staatlichen Zwängen in Form von Recht, Politik und Ökonomie durch zivilgesellschaftlichen Konsens und freiwillige Zustimmung Herrschaft sichert und (re)produziert (Buckel und Fischer-Lescano 2007).

Hegemonie bedeutet, dass die Macht der dominanten Gesellschaftsgruppen sowohl legitimiert wird als auch, dass sie natürlich erscheint - Hegemonie als etwas, das gelebt und woran geglaubt wird. Dabei kommt insbesondere den harmlos anmutenden Handlungen im Alltagsleben von Bürger*innen eine größere makropolitische Bedeutung zu, eben weil sie bestimmten Machtverhältnissen entspringen und diese fortlaufend (re)produzieren (Marchart 2008; Buckel und Fischer-Lescano 2007). Dies ist verkehrspolitisch von Interesse, da es hier erst einmal nicht um großangelegte bundes-, landes-, oder kommunalpolitische Entscheidungen geht, sondern die Politik im Kleinen im alltäglichen Leben auf der Straße stattfindet.

Eine kulturelle Hegemonie ist dabei zwar erst einmal konservierend und wirkmächtig - sie strebt ihr eigenes Bestehen an - aber nie unangefochten stabil 
oder endgültig, sondern muss permanent gegen Herausforderungen verteidigt werden. In diesem Verständnis fungiert Kultur dann sowohl als Stabilisator dominanter Lebensweisen und Leitbilder, indem zum Beispiel bestimmte diskursive Setzungen wie „Deutschland als Autoland“ sowohl materiell wie auch ideologisch verstetigt und normalisiert werden. Aber auch als Motor des Wandels kann Kultur durch (sub)kulturelle Praktiken (z. B. deviante Fahrweisen ${ }^{1}$ oder Raumaneignungen von Radfahrenden) oder veränderte Narrative und Leitbilder (z. B. menschengerechte und lebenswerte Städte) wirken (Müller-Funk 2010; Furness 2010). Dabei werden kulturelle Hegemonien häufig aufrechterhalten und oppositionelle Forderungen entschärft, etwa in Form von Zugeständnissen, durch die ursprünglich als subversiv verstandene Forderungen in die Mehrheitsgesellschaft inkorporiert und somit kommerzialisiert werden (Langemeyer 2009; Marchart 2008; Featherstone 2007; Boltanksi und Chiapello 2006).

\section{Kulturkämpfe als Beginn einer jeden Verkehrswende}

De Facto gibt es die mobilitätskulturelle Hegemonie des „Systems der Automobilität“ (Urry 2004), welches als ,raumkonstituierendes Dispositiv der Moderne" (Manderscheid 2012) zu verstehen ist und durch materielle und kulturell-symbolische Elemente gestützt wird. Dabei ist in Medien und Politik aber immer mehr von einem Kulturkampf die Rede, wobei grundsätzliche Fragen wie „Wem gehört die Straße?“ angesprochen werden und die angesprochene automobile Hegemonie angegriffen wird. Schlagzeilen wie „Die Radfahrer spinnen. Sie treten, spucken, pöbeln. Sie rasen ohne Helm und Licht. Sie klauen uns die Straße" (Drechsler 2017) aus der AutoBild-Ausgabe vom November 2017 zeigen eine emotionale Abwehrhaltung und Entrüstung, die deutlich macht, wie wichtig eine kulturelle Betrachtung sein kann, um aktuelle Tendenzen im Mobilitäts- und Verkehrsbereich angemessen verstehen zu können.

Diese symbolische und emotionale Aufladung von Alltagsverständnissen und -praktiken hat - mobilitätskulturell gefasst - ihren Ursprung in politischen und ökonomischen Entscheidungen sowie Pfadabhängigkeiten wie auch in hegemonialen Narrativen, Diskursen, Weltbildern und Mythen, die diese Machtstrukturen fortlaufend normalisieren, zementieren und (re)produzieren. Die Propagierung einer automobilen Gesellschaft, der Ruf ,Freie Fahrt für freie Bürger' oder die Stigmatisierung von Radfahrenden als ,Kampfradler' müssen

\footnotetext{
${ }^{1}$ Mit devianten Fahrweisen meine ich z. B. das Nicht-Einhalten der StVO wie Rotlichtmissachtungen, das Fahren ohne separate Bremseinrichtungen bei Fixed-Gears oder das NichtBefahren von ausgewiesenen Radwegen.
} 
somit immer als ideologischer Ausdruck bestehender Machtverhältnisse gelesen werden. So wie Radfahrende aufgrund struktureller Subordination quasi dazu gezwungen sind, sich durch subkulturelle und teils deviante Praktiken überhaupt als gleichberechtigte Verkehrsteilnehmende zu konstituieren und im Straßenverkehr zu behaupten (Aldred und Jungnickel 2014; Furness 2010), wurden deren Strategien und Forderungen durch massenmediale Schlagzeilen, verkehrsrechtliche Sanktionen und eine jahrzehntelange politische wie planerische Nichtbeachtung fortlaufend delegitimiert, ausgeschlossen und subordiniert. Dass sich dieses Verständnis (medial, diskursiv, planerisch und politisch) in einem umfassenden Wandel befindet, ist ein deutliches Zeichen dafür, dass sich dem Radverkehr aber auch dem gesamten Umweltverbund ein historisches Möglichkeitsfenster im Kampf um kulturpolitische Deutungsmacht geöffnet hat, weshalb eine Förderung einer neuen Mobilitätskultur besonders hier auf fruchtbaren Boden fällt.

Der Literatur- und Kulturwissenschaftler Wolfgang Müller-Funk (2010, S. 284) schreibt: „Das Wort ,Kulturkampf“ ist zumeist negativ besetzt und hat einen üblen Beigeschmack, erweckt es doch Assoziationen an Repression. (...) Aber Kulturkampf als Streit um Bedeutung gehört zum Alltag von entwickelten zivilen und demokratisch organisierten Gesellschaften. (...) Was gelten soll, was ,bedeutend“ ist, muss ausgehandelt werden“. Dabei zeigt die fortwährend steigende Emotionalität in den verkehrspolitischen Debatten, dass bestehende Strukturen, Überzeugungen, Lebensweisen und Identitäten bereits entscheidend angegriffen wurden und der Kampf um Bedeutungen im vollen Gange ist. Ein ,Kulturkampf ' im Bereich Mobilität und Verkehr ist damit nicht als Problem, sondern als Zeichen einer öffentlichen Anerkennung und Stärkung subordinierter und alternativer Mobilitätskulturen zu lesen und somit notwendiger Bestandteil und Beginn einer jeden Verkehrswende.

\subsection{Integration von Mobilitätskulturen in die Integrierte Verkehrsplanung und -politik}

Im deutschen, wie im angelsächsischen Raum wird ein grundlegender Paradigmenwechsel im Bereich der Verkehrsplanung gefordert (Schwedes und Hoor 2019; Litman 2013; Banister 2008). Aufgrund der Ähnlichkeit der Ansätze in ihrem Fokus auf eine zielorientierte und akteurszentrierte Nachfrageplanung werden diese hier unter dem Begriff Integrierte Verkehrsplanung subsumiert 
(siehe den Beitrag von Schwedes in diesem Band). Die grundlegende Einsicht ist, dass die klassischen technischen und ingenieurswissenschaftlichen Verkehrsplanungsparadigmen (Angebotsplanung und Effizienzsteigerung im Verkehr durch Minimierung von Zeit und Kosten) nicht helfen werden den Verkehr nachhaltiger zu gestalten, sondern - im Gegenteil - den Status quo der autozentrierten Planung weiter zementieren. Ein neues Planungsparadigma der Verkehrsforschung muss deshalb die soziale Sphäre stärker integrieren, sich auf Menschen fokussieren, alternative Verkehrsträger und eine strategische Zielorientierung in die integrierte Betrachtung einbeziehen.

Die integrierte Verkehrsplanung ist somit als ein interdisziplinärer und evidenzbasierter Ansatz zu verstehen, mithilfe dessen die komplexen gesellschaftlichen Verhältnisse angemessen beschrieben und im Rahmen einer nachhaltigen Entwicklung gestaltet werden können. Dabei geht es verkehrspolitisch um eine Aushandlung der unterschiedlichen Interessen sowie einer normativen, politischen, fachlichen und räumlichen Integration, mit dem Ziel eine nachhaltige Verkehrsentwicklung zu fördern (Schwedes und Rammert 2020). Insbesondere mit dem Fokus auf den Menschen, seine Bedürfnisse, Anforderungen und Routinen, sowie der forcierten Verhaltensänderung bei Verkehrsproduzenten und -konsumenten wird eine kulturelle Überarbeitung des bisherigen Planungsverständnisses wichtig (Hoor 2020a).

\section{Konzeption - Grundlagen einer neuen Mobilitätskultur}

Auch wenn Nordeuropa gerne als Paradebeispiel für einen starken Umweltverbund bezeichnet wird, ist die städtische Mobilität auch hier maßgeblich durch das Automobil geprägt und eine Betrachtung der Öffentlichen Mobilität ohne Bezug darauf nur wenig zielführend. Schließlich geht es bei der Gestaltung einer öffentlichen Mobilitätskultur vor allem darum, das Dilemma aufzulösen, dass das privat genutzte Automobil ein Grundbedürfnis nach individueller Mobilität auf Kosten des Gemeinwohls befriedigt (Schwedes 2017; Knoflacher 2009). Während sich im Bereich des Radverkehrs sowie der Radverkehrspolitik die bislang hoffnungsvollsten Veränderungen zeigen, gelang es insbesondere dem öffentlichen Verkehr (ÖV) bislang nicht, sich auf die neuen Mobilitätsbedürfnisse einzustellen und eine grundsätzliche Strukturveränderung herbeizuführen. 


\subsection{Notwendigkeit einer neuen Mobilitätskultur}

Die Gründe, warum es eine neue Mobilitätskultur braucht, sind vielfältig und begründen sich vor allem mit Blick auf die vielfältigen negativen Auswirkungen des MIV. Zu nennen sind u. a. Umweltauswirkungen des Verkehrs in Bezug auf Lärm, Luft, Klima und Flächenverbrauch; Auswirkungen auf die räumlich-zeitliche Organisation von Leben, Arbeit und Freizeit; die Gliederung und Fragmentierung von Städten oder auch die Überführung von öffentlichem Raum in monofunktionale Verkehrsflächen (Becker 2018; SRU 2005; Sheller und Urry 2000; Augé 1995; Illich 1974; Jacobs 1961). Dabei kann vielem durch verkehrliche, wirtschaftliche und politische Entscheidungen und Maßnahmen entgegengewirkt werden, z. B. durch die Internalisierung externer Effekte sowie durch umfassende Umverteilungs- und Umbaumaßnahmen. Um aber auch das Mobilitätsverhalten nachhaltig zu verändern, müssen unterschiedliche Handlungsdimensionen des Menschen betrachtet werden. Dies ist zwingend notwendig, denn während der Anspruch einer nachhaltigen Verkehrsentwicklung gesellschaftlich, politisch, wie planerisch vielfach gewünscht ist, zeigen sich bei der konkreten Umsetzung und Akzeptanz noch Widersprüche: Während beispielsweise ein Großteil der deutschen Bevölkerung findet, dass eine Stadt- und Verkehrsentwicklung mit weniger Autos und mehr Platz für Fuß- und Radverkehr zu einem guten Leben beitragen kann, stehen sie restriktiven Maßnahmen dennoch ablehnend gegenüber (Umweltbundesamt 2017b).

Dabei sind verkehrspolitisch insbesondere die Strategien der Verkehrsverlagerung und -vermeidung bedeutsam für eine nachhaltige Verkehrsentwicklung, wobei technische Maßnahmen der Verkehrseffizienz (z. B. Förderung der Elektromobilität) oft wirtschaftsfreundlicher und politisch opportuner sind und deshalb den verkehrspolitischen Diskurs dominieren (Umweltbundesamt 2019; Schwedes 2018). Hat die Vermeidungsstrategie einen schlechten Ruf, bedarf sie doch eben dieser Restriktionen und eines umfassenden Strukturwandels von Mensch und Gesellschaft, so scheint die Verkehrsverlagerung hingegen mit einer Förderung von ÖV, Rad- und Fußverkehr oder der gemeinschaftlichen Nutzung von Fahrzeugen auszukommen. Allerdings darf man sich auch hier nichts vormachen - eine ,Förderung ' hört sich zwar harmlos an, kann unter den gegebenen Bedingungen aber auch nur durch umfangreiche strukturelle wie mentale Transformations- und Umverteilungsprozesse (z. B. durch umfangreiche Pushund Pull-Maßnahmen) erreicht werden, in dem die bestehende Hegemonie des Automobils massiv eingeschränkt wird ( Holz-Rau 2018). Dies wird bereits am Berliner Mobilitätsgesetz sowie dessen konfliktreicher Entstehungsgeschichte 
deutlich (siehe die Beiträge von Dirk von Schneidemesser und Jens-Holger Kirchner in diesem Band).

Denn gerade in Städten, die innerstädtisch mit einer dichten und kompakten Bauweise über ideale Bedingung für Rad-, Fuß- und öffentlichen Verkehr verfügen, führt die Dominanz des motorisierten Individualverkehrs zwangsläufig zu einer Diskriminierung anderer Transportmittel. So hat Berlin in Bezug auf den Radverkehr von Außenstehenden betrachtet häufig das Image einer fahrradfreundlichen Stadt. Doch während der Radverkehrsanteil in den letzten Jahren deutlich gestiegen ist, wurden die fahrradnahen Infrastrukturen und Dienstleistungen nicht entsprechend ausgebaut. Aufgrund der umfangreichen Arbeit radverkehrspolitischer Initiativen steht die Stadt nun vor einem großen infrastrukturellen Wandel, der im neuen Berliner Mobilitätsgesetz politisch beschlossen worden ist. Dennoch ist das Radfahren in Berlin wegen des langsamen Ausbaus, dem fortwährenden Mangel an sicherer und komfortabler Radinfrastruktur sowie der bestehenden automobilen Dominanz noch immer als eine untergeordnete Praxis zu bewerten, die immer wieder Mut, Geschick und Erfahrung erfordert, was sich u. a. in den schlechten Ergebnissen in Studien zur Radverkehrsfreundlichkeit widerspiegelt (ADFC 2019). Darüber hinaus ist Berlin aber mit seinen hunderten Fahrradläden, den genannten Initiativen, bekannten Messen und Rennen sowie lebhaften und heterogenen Szenen einer der europäischen Hotspots urbaner Radkultur, die bereits einen umfassenden Wandlungsprozess durchlaufen hat. Diese soll im Folgenden kurz skizziert werden, um aus der urbanen Fahrradkultur Anknüpfungspunkte für die Öffentliche Mobilität herzuleiten.

\subsection{Anknüpfungspunkte - Der Radverkehr als Vorreiter der Öffentlichen Mobilität}

Der Radverkehr hat in den letzten 15 Jahren insbesondere in den Städten einen starken Bedeutungswandel erlebt (Nobis 2019). Aus verkehrsplanerischen Gesichtspunkten ist dies verständlich. Im Kontext urbaner Transformationen und den Anforderungen des Klimawandels ist das städtische Radfahren als Form der nachhaltigen Mobilität für die Steigerung/Erhaltung der Lebensqualität und für die Erfüllung von individuellen Bedürfnissen unerlässlich (Pucher und Buehler 2012). Dabei ist das Fahrrad gleichzeitig eines der einfachsten und minimalistischsten Verkehrsmittel, die es überhaupt gibt, aber auch ein technisch hochentwickeltes, vielfältiges und individualisiertes Objekt, das für sehr viele verschiedene Nutzungen und Nutzer*innengruppen geeignet ist (Cox 2015, 2019; Hutchinson 2017; Hadland und Lessing 2016; Vivanco 2013; Horton et al. 2007). 
Menschen fahren aus unterschiedlichsten Gründen Fahrrad, z. B. aus Notwendigkeit, Spaß, Gesundheit, Sport, Überzeugung, aus praktischen Gründen oder um ihre Zugehörigkeit zu einer bestimmten Lebensstilgruppe zu zeigen. Mindestens genauso viele Gründe gibt es, das Rad nicht zu nutzen. All diese unterschiedlichen Bedeutungen sind dabei im Objekt Fahrrad sowie der alltäglichen Praxis des Radfahrens eingeschrieben und werden in Kulturprodukten (z. B. Bücher, Filme, Erzählungen) kommuniziert, ausgehandelt und verfestigt.

Dabei zeichnet sich bei der gesellschaftlichen Normalisierung und den damit einhergehenden Ausprägungen des städtischen Radfahrens ein Wandel ab: Galt das Fahrrad lange Zeit nur als Spiel- und Sportgerät, so ist es heute auch ein Distinktionsmittel großstädtischer Bevölkerungsschichten, Symbol der Verkehrswende und geht mit veränderten Vorstellungen eines guten städtischen Zusammenlebens einher. Der Ursprung dieser Entwicklung ist vor allem in verschiedenen städtischen fahrradbezogenen Subkulturen zu finden wie z. B. der in den 1980er Jahren aufkommenden Fahrradkurier-Szene, die sich stark durch bestimmte Zugehörigkeitssymbole (u. a. Fixed Gears, Kuriertaschen) und Praktiken (u. a. riskanter und selbstbewusster Fahrstil) auszeichnet. Stile und Praktiken von Fahrradkurieren haben in den Nuller-Jahren Eingang in urbane Moden gefunden und sind auch heutzutage noch für einen Großteil der Entwicklung weiterer fahrradbezogener Szenen, wie der aktuellen Fixie-, Rennrad-, oder Cyclocross/Gravel-Szene, relevant (Hoor 2020b; Kidder 2011; Furness 2010; Wehr 2009; Fincham 2007).

Obwohl diese subkulturellen Wurzeln genealogisch zumeist zutreffen, sind städtische Fahrradszenen mittlerweile viel heterogener, kommerzieller und massentauglicher geworden. Illustrieren möchte ich diesen umfassenderen Bedeutungswandel an drei Hollywood-Filmen, welche diese subkulturellen Wurzeln aufgreifen und das Fahrrad als Distinktion und Lifestyle-Element inszenieren: Der Film Premium Rush zum Beispiel handelt von einem Fahrradkurier, der versehentlich in einen Kriminalfall verwickelt wird und spektakulär auf seinem Fahrrad vor Autos durch New York City fliehen muss. Ebenfalls zu nennen ist Tomb Raider, in dem die ausgiebige Eröffnungsszene eine Art Fahrradkurier-Rennen zeigt. Während im Film Quicksilver von 1986 die Figur des Fahrradkuriers den sozialen Abstieg eines ehemals erfolgreichen Bankiers illustriert, werden Fahrradkuriere in Premium Rush und Tomb Raider schließlich zum Helden/ zur Heldin und das Fahrrad zu ihrem mächtigen Werkzeug, analog zum schnellen Auto in den meisten Actionfilmen.

Zusammenfassend ist zu sagen, dass sich in vielen urbanen Fahrradszenen eine starke Ästhetisierung und Lebensstilisierung des Fahrrads zeigt (Hoor 2020b). Subkulturen, die ehemals nur von Profisportler*innen oder 
Fahrradkurier*innen gepflegt wurden, sind heute Teil der Popkultur und gehen mit einer starken Kommerzialisierung einher, z. B.:

- Hipster, die Fixie fahren, Messenger Bags tragen oder sich schicke Rennräder, Gravelbikes und Radklamotten leisten.

- Leute, die sich ihr Fahrrad ins Wohnzimmer hängen, anstatt es auf der Straße stehen zu lassen.

- Produkte aus Fahrradindustrie und Mode, die den modernen Design-Stil mit einer fahrradspezifischen Funktionalität verbinden.

- Fahrradläden, die nicht nur Fahrräder verkaufen, sondern auch als hippes Café, Treffpunkt, Restaurant oder Bar fungieren.

- Medienbeiträge in Zeitungen, Film und Werbung in denen das Fahrrad als cooles und nachhaltiges Fortbewegungsmittel gefeiert und mit neuen Geschichten belegt wird.

Dies sind alles Entwicklungen die nicht radverkehrspolitisch gefördert wurden, sondern sich parallel aus subkulturellen sowie hedonistischen und kommerziellen Interesse gebildet haben und das Fahrrad fortwährend mit neuen Attributen belegen, die immer neue Menschen dafür begeistern.

\subsection{Kritisches Potenzial - Alltagspraktiken und Raumproduktionen}

„Surely it is the supreme illusion to defer to architects, urbanists or planners as being experts or ultimate authorities in matters relation to space." (Henri Lefebvre 1991, S. 95)

Den Alltagspraktiken des Radfahrens und zu Fuß Gehens kommt in mehrfacher Hinsicht eine wichtige Bedeutung für eine öffentliche Mobilitätskultur zu: Neben seinen positiven Effekten für eine nachhaltige Verkehrsentwicklung ist es einerseits als performative Kritik an bestehenden automobilen Raum- und Verkehrsstrukturen zu verstehen, andererseits ist es eine eigene Art der Raumaneignung und -produktion. Etwas idealisiert kann von einer Belebung und Aneignung von Nicht-Orten im Sinne von Marc Augé (1995) und Michel de Certeau (1988) gesprochen werden: Städtische Räume - wie monofunktionale Verkehrsräume werden durch die Praktiken des Radfahrens und des zu Fuß Gehens, ihrer spezifische Wahrnehmung und die damit einhergehenden Erfahrungen neu bespielt 
und konstituiert. Dies wird besonders bei den Critical-Mass-Veranstaltungen ${ }^{2}$ deutlich: „By taking action which temporarily transforms the cityscape to one dominated by bicycles, pedestrians and the sound of human voices, an alternative, sustainable society is being, however temporarily, brought into existence" (Horton 2006, S. 55). Dabei ist die Critical Mass als eine kurzfristige und flüchtige Umsetzung einer Utopie zu lesen, in der sich die Machtverhältnisse auf den Straßen zugunsten des Radverkehrs verschieben (Furness 2005).

Des Weiteren erlauben Radfahren, zu Fuß Gehen und die ÖV-Nutzung einen direkten Kontakt und Austausch mit der städtischen Umwelt und anderen Menschen. Dies wird nicht nur intensiver und sinnlicher wahrgenommen, es wird auch intensiver und sinnlicher genutzt. Radfahrende, die sich mithilfe eigener Muskelkraft weitestgehend ungeschützt fortbewegen, sind deutlich weniger gegenüber ihrer Umgebung abgeschottet als Autofahrer*innen und werden durch die direkte Präsenz sowie der möglichen Interaktion wesentlich ,menschlicher" wahrgenommen als das technische Objekt Automobil (Jensen 2009, 2010; Horton et al. 2007; Spinney 2006). Das Fahrrad produziert eine räumliche Nähe und begünstigt so auch die Wiederbelebung von Innenstädten und öffentlichen Räumen bis hin zur Konstitution von demokratischen Subjekten, die durchs Radfahren zu bürgerschaftlichen Engagement angeregt werden und sich verstärkt mit ihren Nachbarschaften und städtischen Räumen identifizieren (Aldred 2010).

Dabei besteht ein Dilemma der nachhaltigen Verkehrsentwicklung darin, dass sie als „öffentliches, unteilbares Gut kaum gewürdigt wird“ (Schwedes 2017, S. 112). Insbesondere zur Firmierung einer neuen Mobilitätskultur bedarf es einen umfassenden Wertewandel, in dem der öffentliche Raum wieder verstärkt als kollektives Allgemeingut - Urban Commons - verstanden wird, um dass sich von allen Seiten gekümmert werden muss. Dies bedarf allerdings einer verstärkten Identifikation mit dem öffentlichen Raum und ein darauf erwachsendes Verantwortungsbewusstsein seitens der Bevölkerung.

${ }^{2}$ Die Critical Mass ist eine seit 1992 weltweit stattfindende monatliche Veranstaltung zur Förderung des Radverkehrs. Dabei wird in einer großen Gruppe von Radfahrenden durch Städte gefahren und unter dem Motto „We're not blocking Traffic. We ARE Traffic!“ für bessere Radverkehrsbedingungen demonstriert. 


\subsection{Zielbestimmung - Welche neue Mobilitätskultur?}

Die Begriffe multimodale Mobilitätskultur, postfossile Mobilitätskultur, nachhaltige Mobilitätskultur, öffentliche Mobilitätskultur oder auch neue Mobilitätskultur beschreiben alle mehr oder minder dasselbe. Dabei möchte ich aber vor einer ideologischen Homogenisierung warnen: Wenn bereits kleinteiligere Begriffe wie Radverkehr und Radfahren der Vielfalt an Nutzer*innen(gruppen), Praktiken und Bedeutungszuweisungen nicht gerecht werden (siehe Abschn. 3.2), dann wird insbesondere bei Begrifflichkeiten wie Nahmobilität, Umweltverbund, Öffentliche Mobilität sowie den oben genannten von einer Homogenität des Ganzen ausgegangen, welche vermeintliche Gemeinsamkeiten überbetont und schwerwiegende Unterschiede außer Acht lässt. Denn ein Großteil des Selbstverständnisses entspringt aus einem dialektischen und konstitutiven Verhältnis zur aktuellen automobilen Dominanz, welche die inneren Unterschiedlichkeiten fortwährend nivelliert. Die Visionen einer „Radverkehrsinfrastruktur für Alle!“ (ADFC 2018, S. 11), der "Stadt für Morgen“ (Umweltbundesamt 2017a) oder einer umfassenden ,öffentlichen Mobilitätskultur“ sind vielleicht als wünschenswerte Zielsetzung zu verstehen, lassen sich aber durch eine simple sozial- und kulturwissenschaftliche Erkenntnis kritisieren: Jeder Einschluss produziert neue Ausschlüsse!

Darüber hinaus werden der Ausbau von Radinfrastruktur sowie die übergeordneten Leitbilder der ,nachhaltigen Mobilität ' bzw. ,lebenswerten Stadt insbesondere im US-amerikanischen Raum aus einer Perspektive der sozialen Gerechtigkeit stark kritisiert. Radverkehrsförderung sowie die Revitalisierung des öffentlichen Raumes gehe mit einer Wiederentdeckung und Aufwertung der Innenstädte einher und kommt somit insbesondere den ohnehin schon privilegierten Schichten zugute - Verkehrspolitik als eine moderne Form der Klassenpolitik, die bestehende soziale Ungleichheiten (z. B. entlang der klassischen Diskriminationslinien wie Rasse, Klasse und Geschlecht) verfestigt und unter der Legitimation einer vermeintlichen Inklusivität weiter ausweitet. Auch der oben beschriebene Bedeutungsgewinn des Radverkehrs geht mit diesen Problemen einher und reproduziert insbesondere männlich dominierte Sichtweisen aufs Radfahren (MAMILS, ,Strong and Fearless' etc.), wodurch auch eine inklusive Radverkehrsentwicklung aber auch eine sozialgerechte Stadtentwicklung behindert werden kann (Stehlin 2019; Lugo 2018; Lam 2018; Hoffmann 2016; Sheller 2015).

So kann eine Konzeption einer neuen Mobilitätskultur, die ihren eigenem Anspruch gerecht werden möchte, nur gelingen, „wenn es mit einer handlungsleitenden normativen Gerechtigkeitskonzeption verknüpft ist und den 
Zusammenhang von Mobilität und sozialer Exklusion thematisiert" (Daubitz 2018; siehe auch den Beitrag von Daubitz in diesem Band). Eine Aufgabe wird es u. a. sein, eine gemeinsame Zielorientierung hinzubekommen, welche den bildungsbürgerlichen Diskurs mit seiner, Wünsch Dir was!'-Rhetorik übersteigt und auch bislang marginalisierte und nicht am Diskurs teilnehmende Gruppen einbezieht - egal ob potenzielle Verlierer*innen oder Gewinner*innen der Verkehrswende.

Dabei lohnt sich ein Blick auf bisherige Entscheidungsfindungsprozesse - insbesondere was eine öffentliche Meinung angeht, in der bereits stellvertretend um politische Entscheidungen gerungen wird. Die öffentliche Meinung wird durch Resonanz und Zustimmung der Bevölkerung legitimiert, und ist die „Meinung, auf die sich viele öffentlich geeinigt haben" (Hannah Ahrend, zitiert in König 2012, S. 15). So konnte z. B. der Volksentscheid Fahrrad in Berlin auch nur deshalb so erfolgreich sein, weil er dem Berliner Abgeordnetenhaus innerhalb kürzester Zeit über 100.000 Unterschriften für seinen Antrag auf ein Volksbegehren vorlegen konnte - sein politischer Auftrag war damit untermauert, auch wenn es natürlich lautstarke Gegenstimmen gab. Bisherige Umfragen und Partizipationsansätze zur Verkehrswende oder zum Umweltbewusstsein zeigen bereits, dass von einer öffentlichen Meinung gesprochen werden kann, welche das grundsätzliche Ziel der nachhaltigen Verkehrsentwicklung politisch begründet - dies ist durchaus als Erfolg anzusehen, an dem angeknüpft werden kann (Böhm und Herzberg 2020; Umweltbundesamt 2017b).

\section{$3 \quad$ Prozess - Wege zur Öffentlichen Mobilitätskultur?}

„Es ist leichter, Unkraut auszurotten als den Sexismus. Eine ganze Kultur zu transformieren wäre erheblich mühsamer, als einen Fluß zu stauen oder einen Berg abzutragen. " (Terry Eagleton 2009, S. 132)

Vielen Äußerungen im Bereich der Verkehrspolitik und -planung liegt implizit die Annahme zugrunde, dass, weiche" Faktoren wie z. B. die Veränderung von soziokulturellen Verhaltensweisen, Einstellungen und Werte leichter zu verändern seien als die ,harten' Faktoren, wie z. B. Infrastrukturen oder Gesetze. Beim Vergleich dieser Einschätzung mit dem obenstehenden Zitat des Literaturtheoretikers Terry Eagleton zeigt sich die Diskrepanz in den jeweiligen Einschätzungen: Aus kulturtheoretischer Sicht sind Kulturen sehr widerstandsfähig, kontingent und nur ungewiss zu beeinflussen, während Kultur aus Sicht der Mobilitäts- und Verkehrsforschung zumeist eine abgeleitete Größe darstellt. Dabei teile ich die 
kulturtheoretisch fundierte Annahme, dass sich die materielle Seite von Kultur (zum Beispiel die Verkehrsinfrastruktur) leichter bearbeiten lässt als die Geistige. Gerade deshalb ist ein fundiertes Verständnis der geistigen Kulturdimensionen in ihrem Zusammenspiel mit der materiellen Dimension unerlässlich, um den Transformationsprozess zu gestalten.

Dies begründet eine spezifische Förderung des Umweltverbunds durch die zielgerichtete Kombination von restriktiven wie fördernden Maßnahmen bei einem gleichzeitigen Fokus auf Informations-, Evaluations-, Beteiligungs- und Kommunikationsstrategien. Ohne den Einbezug von Letzterem, mit dem Ziel der langfristigen Veränderung dominanter gesellschaftlicher Bilder, Diskurse und Erzählungen, kann - hegemonietheoretisch gesprochen - zwar zentralisierter Zwang (z. B. Fahrverbote), aber niemals anhaltender zivilgesellschaftlicher Konsens geschaffen werden.

\subsection{Verlagerung und Motive der Verkehrsmittelwahl}

Obwohl der Radverkehr einen wichtigen Teil neuer Mobilitätskulturen darstellt, wird an ihm m. E. besonders deutlich, was anderen Verkehrsträgern des Umweltverbundes noch fehlt. Denn der beschriebene Bedeutungswandel des Radfahrens, insbesondere in seinen Zuschreibungen unter jungen Großstädter*innen, ist nicht unbedingt für andere Formen der Öffentlichen Mobilität ersichtlich, auch wenn die Öffentlichkeitskampagnen der BVG der letzten Jahre ein positives Beispiel darstellen. Um letzten Endes dem Automobil den Rang abzulaufen, ist dies aber umso bedeutsamer und müsste von mehreren Akteuren ebenso konsequent umgesetzt werden. Denn „Automobile sind also nicht nur technische Vehikel und Objekte zweckrationalen Handelns und des ökonomischen Kalküls, sondern wesentlich auch sozial und kulturell geprägt" (Schmidt 2018, S. 374). Dabei galt es ,lange Zeit als Symbol für Freiheit und Unabhängigkeit, das sich in die Routinen jedes Einzelnen eingeschrieben hat" (Canzler und Radtke 2019) - das „Auto im Kopf“, wie Andreas Knie (2005) es bezeichnet hat.

Dabei existiert eine große Diversität an verschiedenen Automobilkulturen, wobei das Auto einen Wandel von einem Statussymbol der Bourgeoisie über ein Massenprodukt hin zu einem Produkt differenter Lebensstile der (post) modernen Konsumgesellschaft vollbracht hat (Miller 2001; Featherstone 2004; Schmidt 2018). Zur Einordnung dieser eher sozio-kulturellen Beharrungskräfte auf das individuelle Mobilitätsverhalten hilft ein Blick auf umwelt- und sozialpsychologische Motive der Verkehrsmittelwahl. So lässt sich die Wahl des Verkehrsmittels nämlich nicht nur nach rationalen Gesichtspunkten (z. B. Kosten, 
Geschwindigkeit, Verfügbarkeit, Zeitersparnis) erklären, sondern hängt von komplexeren affektiven und symbolischen Motiven ab (Jakobsson 2007; Steg 2005). Unter affektiven Motiven werden negative Gefühle wie Stress, Unsicherheit oder Anstrengungen aber auch Positive wie Begeisterung, Vergnügen oder Aufregung verstanden. Demgegenüber subsumieren symbolische Motive die Möglichkeiten zur Statusgenerierung und Identifikation. Symbolische Motive erfüllen somit zwei Funktionen: Sie bilden eine Referenz für die soziale Einordnung in eine gesellschaftliche Position und bieten darüber hinaus Möglichkeiten, persönliche Identitäts- und Wertvorstellungen durch unterschiedliche Lebensstile auszudrücken. So kann die Wahl eines bestimmten Verkehrsmittels sowohl aus Prestige- und Statusgründen getroffen werden, als auch Ausdruck eines bestimmten, z. B. umweltfreundlichen Lebensstils sein und Zugehörigkeit $\mathrm{zu}$ einer bestimmten Gruppe oder Wertvorstellungen ausdrücken (Gatersleben 2013; Steg 2005; Steg et al. 2001).

Geht es also um eine Abkehr vom Auto hin zu anderen Verkehrsmitteln der Öffentlichen Mobilität, dann müssen entweder die Gründe der Autonutzung hinfällig werden oder dieselben Attribute durch andere Verkehrsmittel erfüllt werden. Aus meiner eigenen Forschung kenne ich z. B. Personen, die ehemals der Autotuning-Szene angehörten, dieser nun den Rücken gekehrt haben und begeistert dasselbe mit Fahrrädern tun - es ist cooler und billiger. Das Fahrrad hat es bereits zum Statussymbol junger Großstädter*innen geschafft und in manchen Bereichen das Auto abgelöst, nun muss das Radfahren nur noch bequemer und sicherer werden. Eine Ausweitung dieser affektiven und symbolischen Motive auf das zu Fuß Gehen und die ÖV-Nutzung scheint also gewissermaßen notwendig, damit auch diese Verkehrsmittel es mit dem Automobilismus aufnehmen können.

\subsection{Symbolisches Kapital und kulturelle Intermediäre}

Dafür muss sich der ÖV aber zu allererst um die eigene Klientel bemühen und sein eigenes symbolisches Kapital stärken. Der sprachliche Ausdruck der Beförderungsfälle für Fahrgäste im öffentlichen Verkehr wurde lange Zeit für Personen genutzt, die eben keine andere Wahl der privaten Verkehrsmittelnutzung haben (sog. Captives). Dies zeigt das irrtümliche Selbstverständnis, dass der ÖV nur als Rückfallebene für diejenigen funktioniert, die keine Wege und Mittel zur individuellen Mobilität aufbringen können und man sich nicht um weitere Zielgruppen bemühen muss (Dziekan und Zistel 2018).

Auch hat der zeitgenössische Schienenverkehr eher ein eingestaubtes Image: „Beliebt ist sowohl die romantische Wochenendfahrt mit der Tram aus dem 
letzten Jahrhundert wie auch der Ausflug ins Grüne mit der Dampflokomotive oder gar der ,abenteuerliche" Betriebsausflug mit der Draisine" (Schwedes 2014, S. 241). Dieser historische Technikfetischismus scheint jedoch nicht massenkompatibel zu sein. Grobschlächtig gesagt, taugt technisches Fachwissen zum ÖV nicht unbedingt als kulturelles Kapital: Zeugt die Kenntnis und Unterscheidung von Standert, 8 bar und Schindelhauer oder von Dura Ace und Ultegra von Stil und Fachwissen unter sportiven (Berliner) Radfahrer*innen, so erscheint mir die Kenntnis über unterschiedliche S-Bahn-Baureihen (z. B. ,Cola-Dose" und ,Taucherbrille") dagegen ziemlich nerdig. Um nicht falsch verstanden zu werden: Nerdig wirkt technisches Fachwissen für Außenstehende ab einem bestimmten Punkt immer, der Unterschied liegt darin, dass das Fachwissen über Fahrräder in einem größeren Maßstab Gegenstand von Alltagsgesprächen und -beobachtungen, Lifestyle-Entscheidungen, Werbekampagnen oder Kulturprodukten ist und somit stärker verbreitet, normalisiert und verstanden wird. Das Fahrrad fungiert dabei häufig als Anzeiger für etwas anderes, beispielsweise eines nachhaltigen, urbanen Lebensstils oder für den Ausflug ins Grüne. Mit Fahrrädern können sich Leute identifizieren, ihre Geschmackspräferenzen ausdrücken und sich von anderen abgrenzen. So ist es beispielsweise ein Unterschied im Statement sowie in der Außenwahrnehmung ob ich Fixie oder Hollandrad fahre diese kulturelle Distinktion gelingt mir aber nicht, wenn ich die Regionalbahn anstatt der S-Bahn nehme.

Für diese Bedeutungszuschreibungen sind insbesondere bestimmte kulturelle Intermediäre wichtig, eben um kulturelle Veränderungsprozesse frühzeitig aufzuspüren und für eine breitere Gesellschaft kommerziell aufzubereiten. Am Fallbeispiel des städtischen Radverkehrs wird deutlich, wie bestimmten Akteur*innen in der Fahrradszene (z. B. Kurier*innen, Hobby- und Amateursportler*innen, Hipster) durch ihre Produktion, Distribution und Vermarktung von Produkten, Dienstleistungen und Lebensstilen eine Funktion als "neoliberale Rolemodels“ zugeschrieben werden kann, wie Sebastian Schweer (2014, S. 167) sie auch Skateboard-Fahrer*innen attestiert. Grundlage hierfür ist ein Schwebezustand „Zwischen urbaner Rebellion und neoliberalem Selbstentwurf“ (ebd.), zwischen Szene-Kenntnis und Marketing-Geschick (Engelmann 1999). Insbesondere hierfür sind aber die Möglichkeiten zur Individualisierung, zur Selbstdarstellung und zur Identifikation die wichtigsten Voraussetzungen, welche bei den Individualverkehrsmitteln augenscheinlich stärker ausgeprägt sind.

Eine neue Mobilitätskultur hat also nach meiner Beobachtung, abgesehen vom Radverkehr, ein gewisses Image- als auch Rolemodel-Problem: Der Öffentlichen Mobilität fehlt es bislang an diesen individuellen, wie kollektiven Ausgestaltungsund Identifikationsmöglichkeiten, an symbolischen Kapital, sowie an jemanden, 
der diese authentisch verkörpern und politisch, wirtschaftlich, planerisch, wie medial wirksam in Wert setzen kann. Dies ist einerseits als Defizit zu werten, eröffnet aber anderseits auch neue Möglichkeiten, eben weil bestehende Kodierungen noch nicht zu stark von bestimmten Gruppierungen vorbelastet sind (siehe Beispiel Fahrradkultur in Abschn. 3.2). Der ausstehende Imagewandel der Öffentlichen Mobilität könnte also noch dazu genutzt werden, ihn von vornherein inklusiver, nachhaltiger und gerechter zu gestalten.

\subsection{Neue Narrative, Ästhetisierungen, Kulturprodukte und mediale Repräsentationen}

„Uns fehlen Bilder, positive Visionen und Geschichten einer neuen Mobilitätskultur. Es fehlt die innere Landkarte eines Kontinents zu dem wir uns hingezogen fühlen, weil seine Versprechen attraktiver sind als das Erleben der Gegenwart" (Stephan Rammler 2018, S. 38)

In Rückbezug auf das Beispiel städtischer Fahrradkulturen (Abschn. 3.2) möchte ich auf folgende Punkte eingehen, die für den Bedeutungsgewinn des Radverkehrs von großer Wichtigkeit waren, die aber im Bereich der Öffentlichen Mobilität noch herzustellen sind: Neue Narrative, mediale Repräsentationen, Kulturprodukte und eine Ästhetisierung von Objekten und Praktiken. Um es in Anlehnung an das obenstehende Zitat von Stephan Rammler zu sagen, fehlt uns momentan noch die konkrete Vorstellung und Erfahrung, wie eine öffentliche Mobilitätskultur ausschauen könnte und wie sich das Leben dann anfühlen würde. Dabei gibt es viele einzelne Maßnahmen und Ideen, aber diese wirken allesamt noch etwas isoliert und reduktionistisch. Denn holistische Visionen einer neuen Mobilitätskultur müssen sowohl der Komplexität des Alltagslebens, als auch den divergierenden Zielgruppen, Interessen, Bedürfnissen und Zugängen gerecht werden: Die Gleichung, weniger Autos + mehr Radverkehr $=$ lebenswerte Stadt" wie sie beispielsweise gerne von Radverkehrsinitiativen vollführt wird, wird dieser Komplexität nicht gerecht.

Dabei ist es durchaus fraglich, ob es solche verkehrsmittelübergreifenden, holistischen und wirkmächtigen Bilder, Narrative und Visionen für eine neue Mobilitätskultur überhaupt geben kann oder ob diese nicht zwangsläufig immer partiell, perspektivisch und spezifisch sein müssen. Um dies zu untersuchen und etwaige Veränderungen ablesen zu können, wäre ein Blick auf die unterschiedlichen Kulturprodukte und medialen Repräsentationen wichtig, die mit unterschiedlichen Verkehrsmitteln in Verbindung gebracht werden und auch 
unterschiedliche Mobilitätskulturen repräsentieren. Denn insbesondere diese Kulturprodukte und medialen Repräsentationen sind wichtig, um bestimmte Erzählungen, Lesarten, Bilder, Symbole und Verständnisweisen hervorzubringen, $\mathrm{zu}$ verbreiten und einem breiteren gesellschaftlichen Kontext $\mathrm{zu}$ verfestigen, sei es in Literatur, Film, Musik, Zeitung oder den Sozialen Medien: Sei es der VW Samba als Hippie-Ikone in Verbindung mit dem Woodstock-Sound oder das Fixed-Gear, die Kuriertasche und Punkrock-Musik für Fahrradkuriere - was wären die stereotypischen und unverkennbaren Darstellungen einer Öffentlichen Mobilität, resp. einer neuen Mobilitätskultur?

Bei alledem ist nicht zu vergessen, dass unsere Phantasie und Begierde vor allem durch ästhetische Zurschaustellungen angeregt werden. Durch den Überfluss an Bildern und Zeichen, welche die städtische Umwelt ästhetisieren, ist die Zeichenfunktion von Gebrauchsgegenständen oft wichtiger als ihre technische Funktion, also ihr Gebrauchswert (Chambers 1986; Jameson 1984; Baudrillard 1968). Ein klassisches Beispiel ist der Kauf eines teuren Fahrzeugs: Ein Teil des Wertes eines Automobils ist auf seine Funktionalität und seine Materialkosten zurückzuführen. Dennoch werden sehr teure Autos vor allem aus gesellschaftlichen Prestigegründen - ihrem Zeichenwert - konsumiert. Diese individualistischen und symbolischen Motive, wie Sichtbarkeit, Selbstdarstellung, Distinktion und Lebensstile, sind wichtig für die Konstitution öffentlicher Mobilitätskulturen, insbesondere wenn es darum geht junge Menschen frühzeitig einzubeziehen (Umweltbundesamt 2019). Denn, um es mit der Automobilität aufzunehmen, müssen auch soziale Anerkennungskämpfe ausgefochten werden und dies funktioniert vor allem über die Ästhetisierung vornehmlich funktionaler Bereiche, wie es am Beispiel der Mode selbstverständlich ist, aber eben auch für die Automobilität und zunehmend für den Radverkehr gilt.

Um dies zu erreichen, muss einerseits der städtische Raum den (ästhetischen) Bedürfnissen und Eigenlogiken des Umweltverbundes angepasst werden (Forsyth und Krizek 2011; Gehl 2010). Andererseits bedarf es neuer Leuchtturmprojekte, welche analog zur Autobahn der autogerechten Stadt oder auch dem Boulevard bzw. dem Bahnhof für die Moderne, als Ikonen für eine öffentliche Mobilitätskultur gelten können, den Wandel symbolisch vorwegnehmen und uns die Richtung weisen. Betrachtet man aber Verkehrsmittel nur als reine Transportmittel, um schnell, sicher und bequem von A nach B zu kommen, dann verkennt man deren kulturellen Wert (Jensen 2009, 2010). Ohne diese identitätsstiftenden Funktionen (sei es für Städte, Gruppen oder Individuen) wird die notwendige Individualisierung und Attraktivierung des öffentlichen Kollektivverkehrs kulturell nur schwer zu erreichen sein. 
Darüber hinaus müssen sich kulturelle Werte ändern: So könnte sich eine Kultur des Gefahren-Werdens, welche als ehemaliger bourgeoiser Luxus vom Narrativ der individuellen Mobilität, des Selber-Fahrens, abgelöst worden ist, vielleicht wieder neu firmieren. Die Idee gefahren zu werden und die Fahrzeit für sinnvollere Tätigkeiten zu nutzen wird nämlich als eines der wichtigsten Argumente fürs autonome Fahren gehandelt (Cyganski et al. 2015) - während es für ÖV-Nutzer*innen schon heute zur Selbstverständlichkeit geworden ist.

\subsection{Neue Kulturtechniken und Routinen}

„Wir haben [fast] alle das Autofahren gelernt. Irgendwann einmal ist uns diese Kulturtechnik zur Selbstverständlichkeit geworden. Wir denken nicht daran, wie wir unsere Beine zwischen Gaspedal, Kupplung und Bremspedal hin- und herbewegen, wir schalten automatisch und schauen wie von selbst in den Rückspiegel.“ (Wolfgang Müller-Funk 2010, S. 17)

Wie in der obenstehenden Beschreibung ersichtlich, vollzieht sich ein Großteil von dem, was wir unter Kultur verstehen, nicht bewusst oder explizit, sondern unbewusst und implizit. Es ist eine zutiefst verborgene, internalisierte und habitualisierte Kulturtechnik, die nur deshalb funktioniert, weil sie eben automatisch abläuft und keine mentale, sensorische und körperliche Überforderung hervorruft. Eine Veränderung von gewohnten Verkehrsregeln und -infrastrukturen (z. B. durch Baustellen) aber eben auch der Wechsel zu anderen Verkehrsmitteln besitzt deshalb ein so großes Irritations- und Überforderungspotenzial, weil das kulturell Unbewusste dann aktiv angepasst und überarbeitet werden muss (Müller-Funk 2010, S. 17 f.).

Um neue Mobilitätskulturen erfolgreich im Alltagshandeln von Menschen zu verankern, müssen die gewünschten Mobilitätspraktiken nämlich von Grund auf erlernt, gelebt und zur Selbstverständlichkeit werden. Ich möchte dies an einem einfachen Beispiel verdeutlichen. Wenn man seinen Arbeitsweg vorher noch nie mit dem Fahrrad bestritten hat, und nun damit beginnt, dann wird man zwangsläufig mit bestimmten Herausforderungen konfrontiert werden, wie z. B.:

- Einem fehlt die realistische Vorstellung davon, wie lange ein Weg dauert, wo man am besten lang fahren muss, wie man sich unterwegs navigiert, was man bei welchem Wetter anzieht.

- Man wird sich unweigerlich verfahren, zu spät oder verschwitzt zur Arbeit erscheinen, sich einen Platten fahren oder sich gar verletzen. 
- Man wird sich über die unangemessene und stückhafte Radinfrastruktur sowie die für den Autoverkehr optimierten Verkehrsprozesse ärgern.

- Man wird in Konflikt mit anderen Verkehrsteilnehmenden treten - wie von Autos angehupt, bedrängt oder geschnitten zu werden - atmet Abgase ein, schlängelt sich zwischen Autos hindurch oder weicht irgendwann genervt auf den Gehweg aus.

Solche oder ähnliche Erlebnisse sind unausweichlich und werden immer wieder als Universalien des Radfahrens beschrieben (Fournel 2019). Um dennoch ein gewünscht multimodales und flexibles Mobilitätsverhalten $\mathrm{zu}$ ermöglichen und Leute langfristig aus dem Auto zu holen, müssen bestimmte Praktiken und Routinen bereits frühzeitig in den Sozialisationsprozess von Kindern und Jugendlichen verankert werden (viele Kinder wissen nicht, wie man Bus oder Fahrrad fährt oder kennen ihren eigenen Schulweg nicht). Zudem müssen auch die unausweichlichen Probleme und Konflikte offen angesprochen, thematisiert und gemildert werden, damit sich neue Routinen einstellen können. Dafür muss die Verkehrsplanung ihren ingenieurs- und wirtschaftswissenschaftlichen Stand weiter aufbrechen sowie verstärkt inter- und transdisziplinär mit anderen Fachbereichen wie der Bildungs- und Sozialisationsforschung, der Stadtentwicklung und Wohnungspolitik, der Gesundheitsforschung, aber auch mit Kultureinrichtungen und Sportvereinen zusammenarbeiten. Denn eines scheint mir offensichtlich: Einmal Nutzer*in eines bestimmten Verkehrsmittels, immer Nutzer*in dieses Verkehrsmittels - es muss darum gehen, diese Beharrungskräfte im Falle der Automobilnutzung aufzubrechen, den Übergang zur Nutzung anderer Verkehrsmittel zu gestalten und die ersten Hürden auf dem Weg zu neuen Routinen aus dem Weg zu räumen.

\section{Fazit}

An dieser Stelle möchte ich betonen, dass Kultur nie referenzlos oder ahistorisch ist. Insbesondere für einen kulturellen Wandel braucht es Ursprünge und Bruchlinien wie bestimmte Alltagspraktiken und Bedeutungen, die bereits heute gelebt, produziert und verfeinert werden. Das bedeutet, dass diese erkundet und verstanden werden müssen, was wiederum die Notwendigkeit einer verstärkten qualitativen und möglicherweise auch ethnographisch oder kulturwissenschaftlich fundierten Mobilitätsforschung betont, welche subjektive Wahrnehmungen, kollektive Bedeutungszuschreibungen, mediale Repräsentationen und alltägliche Praktiken zu erkunden und verstehen versucht. 
Aus genannten Gründen kann es nicht Ziel dieses Beitrages sein, eine konkrete Vorstellung zu konstruieren, wie eine neue Mobilitätskultur aussehen könnte. Dies können z. B. wissenschaftliche Methoden der Zukunftsforschung eruieren und verschiedene Entwicklungspfade aufzeigen (siehe den Beitrag von Christina Wolking in diesem Band). Stattdessen möchte ich lieber noch einmal die Kontingenz, Pluralität und Widersprüchlichkeit gesellschaftlicher Prozesse und Entwicklungen betonen, die eine verkehrsplanerische Steuerung immer wieder überraschen und konterkarieren können. Dabei muss eine neue Mobilitätskultur mehrere gegenläufige und vermeintlich unvereinbare Tendenzen zusammendenken: Solidarität und Kollektivität genauso wie Eigensinn und Individualität; Nutzen wie Besitzen; Regulierung des Autoverkehrs ebenso wie eine Attraktivierung des Umweltverbundes; eindeutige politische Setzungen ebenso wie die Beteiligung der Bevölkerung etc.

Denn jeden Tag werden neue Bilder, Narrative und Ideen produziert, wie eine nachhaltige Mobilität der Zukunft aussehen könnte. Sei es von Bürger*innen, Initiativen, Unternehmen, Wissenschaft oder Politik. Sei es eine überdachte Radstrecke, eine rigorose Umverteilung des Straßenraumes oder Technikvisionen von autonomen Autos und Flugtaxis. Und ebenso vielfältig unser Leben ist, so unterschiedlich sind auch unsere Vorstellungen einer neuen Mobilitätskultur. Wir müssen bereit sein, diesen neuen Vorstellungen und Ideen einen Platz zu geben, sie gemeinschaftlich auszuhandeln, zu legitimieren und zu tragen - materiell wie symbolisch.

Auf beiden Ebenen bedarf es einer umfassenden Kehrtwende von der Automobilität: Im extremen Fall das Auto als ,the new tobacco', dem man sich kollektiv entledigen muss, welches mit Warnhinweisen versehen wird und für dessen Nutzung man sich eigentlich schämen sollte. ${ }^{3}$ Stattdessen bedarf es einer Kultur der Öffentlichkeit, des Miteinanders, der Verantwortung und der Solidarität. Das heißt aber nicht, dass es keinen Platz mehr für Individualität, Abgrenzung und Autonomie geben wird - diese Bedürfnisse können weiterhin erfüllt werden, wie der Bedeutungsgewinn des Fahrrads eindrücklich zeigt. Und auch zukünftig werden Menschen ihr Auto lieben und ihre Autokultur pflegen, problematisch wird erst die strukturell verankerte und unhinterfragte Rationalität und Normalisierung mit welcher der Automobilismus einseitig - auch in Städten - gerechtfertigt wird.

\footnotetext{
${ }^{3}$ Dies soll nicht schmälern, dass das Auto insbesondere im ländlichen Raum faktisch eine essentielle und notwendige Zugangsmöglichkeit zu Bereichen der Grundversorgung und gesellschaftlicher Teilhabe darstellt (Kutter 2016).
} 
Zusammenfassend gesagt ist die Firmierung einer neuen Mobilitätskultur als gesamtgesellschaftliche Aufgabe anzusehen, die meines Erachtens eine Art der doppelten (Re)Regulierung erfordert: Einerseits die strikte öffentliche Verwaltung und Regulierung der Automobilität durch infrastrukturelle und ordnungspolitische Maßnahmen, wie einer flächendeckenden Geschwindigkeitsbegrenzung, dem Parkraummanagement oder einer Flächenumverteilung, wie auch der symbolischen und emotionalen Degradierung - Autofahren im städtischen Alltag muss auf allen Ebenen unattraktiv werden. Gleichzeitig ist eine stärkere Förderung und Regulierung des Umweltverbunds notwendig, wie eine Entkriminalisierung des Schwarzfahrens, digitale Ticketinglösungen oder am besten direkt den kostenlosen ÖPNV; eine radikale Überarbeitung der StVO, um den Bedürfnissen und Alltagspraktiken von Radfahrenden und zu Fuß Gehenden gerecht zu werden etc. - dies alles gepaart mit infrastrukturellen Maßnahmen, wie eine flächendeckende Barrierefreiheit zum ÖV sowie ausreichend breite und attraktive Fuß- und Gehwege. Dabei können Mut und Fehlertoleranz, aber auch eine partizipative Einbindung, sowie Reallabore als Versuchsorte dabei helfen, öffentliche Mobilitätskulturen vor Ort konkret erfahrbar und plastisch zu machen, Akzeptanz zu schaffen und die betroffenen Menschen sowie andere Stakeholder langfristig an Bord zu holen.

Dazu kann auch die Verkehrsplanung und Mobilitätsforschung einen großen Beitrag leisten, indem sie einerseits ziel- und akteursorientierte Maßnahmen aufdeckt, moderiert, operationalisiert und umsetzt, sowie anderseits nach aktuellen Anknüpfungspunkten und Veränderungen forscht - denn wer nicht nach einer neuen Mobilitätskultur sucht, der wird sie auch nicht finden.

\section{Literatur}

ADFC. 2019. Auswertung Stadtgrößenklasse: $>500.000$ Einwohner. ADFC FahrradklimaTest. Berlin: Allgemeiner Deutscher Fahrrad-Club e. V.

ADFC. 2018. So geht Verkehrswende - Infrastrukturelemente für den Radverkehr. Berlin: Allgemeiner Deutscher Fahrrad-Club e. V.

Ahrend, Christine, S. Daubitz, O. Schwedes, U. Böhme, M. Herget. 2013. Kleiner Begriffskanon der Mobilitätsforschung. IVP Discussion Paper 1 (Alte Version), Berlin.

Aldred, Rachel und K. Jungnickel. 2014. Why culture matters for transport policy: the case of cycling in the UK. Journal of Transport Geography 34: 78-87.

Aldred, Rachel. 2010. , On the outside': constructing cycling citizenship. Social \& Cultural Geography 11 (1): 35-52.

Augé, Marc. 1995. Non-Places. Introduction to an Anthropology of Supermodernity. London/New York: Verso Books.

Banister, David. 2008. The sustainable mobility paradigm. Transport Policy 15: 73-80. 
Baudrillard, Jean. 1968. The System of Objects. London: Verso Books.

Becker, Udo. 2018. Verkehr und Umwelt. In Verkehrspolitik: Eine interdisziplinäre Einführung, Hrsg. Oliver Schwedes. Wiesbaden: Springer.

Böhm, Birgit und C. Herzberg. 2020. Bürgergutachten „Mehr Kiez - Weniger Auto: Neue Mobilitätskonzepte für Stadtviertel in Berlin “. Berlin: Mobility2Grid.

Boltanksi, Luc und E. Chiapello. 2006. Der nеuе Geist des Kapitalismus. Konstanz: UVK Verlagsgesellschaft $\mathrm{mbH}$.

Buckel, Sonja und A. Fischer-Lescano. 2007. Hegemonie gepanzert mit Zwang. Zivilgesellschaft und Politik im Staatsverständnis Antonio Gramscis. Baden-Baden: Nomos Verlag.

Canzler, Weert und J. Radtke. 2019. Der Weg ist das Ziel: Verkehrswende als Kulturwende. Oder: Zur schwierigen Entwöhnung vom Auto. Aus Politik und Zeitgeschichte 43: 33-38.

Certeau de, Michel. 1988. Kunst des Handelns. Berlin: Merve Verlag.

Chambers, Ian. 1986. Popular Culture. The Metropolitan Experience. London/New York: Routledge.

Cox, Peter. 2019. Cycling: A Sociology of Velomobility. London: Routledge.

Cox, Peter. 2015. Cycling Cultures. Chester: University of Chester Press.

Cyganski, Rita, E. Fraedrich und B. Lenz. 2015. Travel-time valuation for automated driving: A use-case-driven study. Proceedings of the 94th Annual Meeting of the Transportation Research Board, 11-15. Januar 2015, Washington, USA.

Daubitz, Stephan. 2018. Mobilität und Exklusion. In Verkehrspolitik: Eine interdisziplinäre Einführung, Hrsg. Oliver Schwedes. Wiesbaden: Springer.

Deffner, Jutta, K. Götz, S. Schubert, C. Potting, G. Stete, A. Tschann und W. Loose. 2006. Schlussbericht zu dem Projekt „Nachhaltige Mobilitätskultur“. Entwicklung eines integrierten Konzepts der Planung, Kommunikation und Implementierung einer nachhaltigen, multioptionalen Mobilitätskultur. Frankfurt am Main: ISOE.

Divall, Colin und G. Revill. 2005. Cultures of transport: representation, practice and technology. Journal of Transport History 26 (1): 99-111.

Drechsler, Tom. 2017. Die Radfahrer spinnen. Sie treten, spucken, pöbeln. Sie rasen ohne Helm und Licht. Sie klauen uns die Straße. AutoBild 40.

Dziekan, Katrin und Meinhard Zistel. 2018. Öffentlicher Verkehrs. In Verkehrspolitik: Eine interdisziplinäre Einführung, Hrsg. Oliver Schwedes. Wiesbaden: Springer.

Eagleton, Terry. 2009. Was ist Kultur? München: Verlag C.H. Beck oHG.

Engelmann, Jan. 1999. Think different. Eine unmögliche Einleitung. In Die kleinen Unterschiede. Der Cultural Studies Reader, Hrsg. Jan Engelmann. Frankfurt a. M.: Campus Verlag.

Featherstone, Mike. 2007. Consumer Culture and Postmodernism. Los Angeles/New Dehli/ Singapore: Sage Publications.

Featherstone, Mike. 2004. Automobilities. An Introduction. Theory, Culture \& Society 21 (4-5): 1-24.

Fincham, Ben. 2007. Bicycle Messengers: Image, Identity and Community. In Cycling and Society, Hrsg. Dave Horton, P. Rosen and P. Cox, 179-195. Hampshire: Ashgate Pubslishing.

Forsyth, Ann und K. Krizek. 2011. Urban Design: Is there a Distinctive View from the Bicycle? Journal of Urban Design 16 (4): 531-549. 
Fournel, Paul. 2019. Need for the Bike. London: Pursuit Books.

Furness, Zack. 2005. Biketivism and Technology: Historical Reflections and Appropriations. Social Epistemology 19 (4): 401-417.

Furness, Zack. 2010. One Less Car. Philadelphia, Pennsylvania: Temple University Press.

Gatersleben, Birgitta. 2013. Psychological Motives for Car Use. In Handbook of Sustainable Travel, Hrsg. Tommy Gärling, D. Ettema und M. Friman, 85-94. Dordrecht: Springer.

Gehl, Jan. 2010. Cities for People. Chicago: Island Press.

Götz, Konrad, Jutta Deffner und Thomas Klinger. 2016. Mobilitätsstile und Mobilitätskulturen - Erklärungspotenziale, Rezeption und Kritik. In Handbuch Verkehrspolitik, Hrsg. Oliver Schwedes. Wiesbaden: Springer.

Grieger, Manfred. 2019. Kleine Geschichte des Automobils in Deutschland. Aus Politik und Zeitgeschichte 43: 19-26.

Gramsci, Antonio. 1992. Gefängnishefte. Band 4. Hamburg: Argument.

Hadland, Tony und H.-E. Lessing. 2016. Bicycle Design: An Illustrated History. Cambridge, MA: MIT Press.

Hall, Stuart. 2004. Ideologie, Identität, Repräsentationen. Ausgewählte Schriften 4. Hamburg: Argument.

Hall, Stuart. 2000. Cultural Studies. Ein politisches Theorieprojekt. Ausgewählte Schriften 3. Hamburg: Argument.

Hoffmann, Melody L. 2016. Bike Lanes Are White Lanes. Lincoln: University of Nebraska Press.

Hoor, Maximilian. 2020a. Mobilitätskulturen. Über die Notwendigkeit einer kulturellen Perspektive der Verkehrsplanung. IVP Discussion Paper 2020 (1), Berlin.

Hoor, Maximilian. 2020b. The bicycle as a symbol of lifestyle, status and distinction. A cultural studies analysis of urban cycling (sub)cultures in Berlin. Applied Mobilities.

Holz-Rau, Christian. 2018. Verkehr und Verkehrswissenschaft. In Verkehrspolitik: Eine interdisziplinäre Einführung, Hrsg. Oliver Schwedes. Wiesbaden: Springer.

Horton, Dave, R. Rosen, P. Cox. 2007. Cycling and Society. Hampshire/Burlington: Ashgate Publishing Limited.

Horton, Dave. 2006. Environmentalism and the bicycle. Environmental Politics 15 (1): 41-58.

Hutchinson, Michael. 2017. Re:Cyclists: 200 Years on two wheels. London/New York: Bloomsbury.

Illich, Ivan. 1974. Energy and Equity. New York: Harper \& Row.

Institute for Mobilities Research (ifmo). 2013. Megacity Mobility Culture: How Cities Move on in a Diverse World. Wiesbaden: Springer VS.

Jacobs, Jane. 1961. The death and life of great American cities. New York: Random House.

Jakobsson, Cecilia. 2007. Instrumental motives for private car use. In Threats to the Quality of Urban Life from Car Traffic: Problems Causes, and Solutions, Hrsg. Tommy Gärling und L. Steg, 205-217. Amsterdam: Elsevier.

Jameson, Frederic. 1984. "Postmodernism, or the Cultural Logic of Late Capitalism," New Left Review 146: 53-92.

Jensen, Ole B. 2010 Negotiation in Motion: Unpacking a Geography of Mobility. Space and Culture 13 (4): 389-402. 
Jensen, Ole B. 2009. Flows of Meaning, Cultures of Movements - Urban Mobility as Meaningful Everyday Life Practice. Mobilities 4 (1): 139-158.

Kidder, Jeffrey L. 2011. Urban Flow: Bike Messengers and the City. Ithaca, NY: Cornell University Press.

Klinger, Thomas, J., R. Kenworthy und M. Lanzendorf. 2013. Dimensions of urban mobility cultures - a comparison of German cities. Journal of Transport Geography 31: 18-29.

Knie, Andreas. 2005. Das Auto im Kopf. Die Auswirkungen moderner Verkehrsinfrastruktur auf die Mobilität der Bevölkerung im ländlichen Raum. Zeitschrift für Agrargeschichte und Agrarsoziologie 1: 59-69.

Knoflacher, Hermann. 2009. Virus Auto. Die Geschichte einer Zerstörung.Wien: Verlag Carl Ueberreuter.

König, Tim. 2012. In guter Gesellschaft? Einführung in die politische Soziologie von Jürgen Habermas und Niklas Luhmann. Wiesbaden: Springer VS.

Kutter, Eckhard. 2016. Siedlungsstruktur und Verkehr: Zum Verständnis von Sachzwängen und individueller Verkehrserreichbarkeit in Stadtregionen. In Handbuch Verkehrspolitik, Hrsg. Oliver Schwedes. Wiesbaden: Springer.

Langemeyer, Ines. 2009. Antonio Gramsci: Hegemonie, Politik des Kulturellen, geschichtlicher Block. In Schlüsselwerke der Cultural Studies, Hrsg. Andreas Hepp, F. Krotz, T. Thomas, 72-82. Wiesbaden: Springer VS.

Lefebvre, Henri. 1991. The production of space. Oxford and Cambridge, MA: Blackwell.

Litman, Todd. 2020. Autonomous Vehicle Implementation Predictions. Implications for Transport Planning, Victoria Transport Policy Institute, https://www.vtpi.org/avip.pdf. Zugegriffen: 19. Juni 2020

Litman, Todd. 2013. The New Transportation Planning Paradigm. ITE Journal 8 (6): 20-27.

Lugo, Adonia. 2018. Bicycle/Race. Transportation, Culture, \& Resistance. Portland, Oregon: Microcosm Publishing.

Lam, Tiffany. 2018. Cycling London: An Intersectional Feminist Perspective. Masterarbeit. LSE Cities, London.

Manderscheid, Katharina. 2012. Automobilität als raumkonstituierendes Dispositiv der Moderne. In Die Ordnung der Räume. Henning Füller und B. Michel, 145-178. Münster: Westfälisches Dampfboot.

Marchart, Oliver. 2008. Cultural Studies. Konstanz: UVK Verlagsgesellschaft mbH.

Miller, Daniel. 2001. Car Cultures. Oxford: Berg Publisher.

Mitchell, Don. 2000. Cultural Geography. A Critical Introduction. Oxford/Malden, Mass: Blackwell Publisher Inc.

Müller-Funk, Wolfgang. 2010. Kulturtheorie. Tübingen: Narr francke Attempto Verlag.

Nobis, Claudia. 2019. Mobilität in Deutschland - MiD Analysen zum Radverkehr und Fußverkehr. Studie von infas, DLR, IVT und infas 360. Bonn, Berlin: Bundesministeriums für Verkehr und digitale Infrastruktur.

Nünning, Ansgar und Vera Nünning. 2008. Kulturwissenschaften: Eine multiperspektivische Einführung in einen interdisziplinären Diskussionszusammenhang. In Einführung in die Kulturwissenschaften, Hrsg. Ansgar Nünning und V. Nünning, 1-18. Stuttgart/Weimar: J.B. Metzler.

Pucher, John und R. Buehler. 2012. City Cycling. Cambridge, MA: MIT Press. 
Rammler, Stephan. 2018. Verkehr und Gesellschaft. Verkehrspolitik als Mobilitätsdesign. In Verkehrspolitik: Eine interdisziplinäre Einführung, Hrsg. Oliver Schwedes. Wiesbaden: Springer.

Reents, Edo. 2019. Wider den Fetisch Auto. Aus Politik und Zeitgeschichte 43: 08-11.

Rudolph, Frederic, T. Koska und C. Schneider. 2018. Verkehrswende für Deutschland. Der Weg zur $\mathrm{CO}_{2}$-freier Mobilität bis 2035. Wuppertal: Wuppertal Institut für Klima, Umwelt, Energie im Auftrag von Greenpeace.

Schmidt, Gert. 2018. Automobil und Automobilismus. In Verkehrspolitik: Eine interdisziplinäre Einführung, Hrsg. Oliver Schwedes. Wiesbaden: Springer.

Schwedes, Oliver und Alexander Rammert. 2020. Was ist Integrierte Verkehrsplanung. Hintergründe und Perspektiven einer am Menschen orientierten Planung. IVP Discussion Paper 2020 (2).

Schwedes, Oliver und M. Hoor. 2019. Integrated Transport Planning: From Supply- to Demand-Oriented Planning. Considering the Benefits. Sustainability 11, Special Issue "Sustainable Mobility: Interdisciplinary Approaches".

Schwedes, Oliver, Stephan Daubitz, Alexander Rammert, Benjamin Sternkopf und Maximilian Hoor. 2018. Kleiner Begriffskanon der Mobilitätsforschung (Neuauflage). IVP Discussion Paper 1, Berlin.

Schwedes, Oliver. 2020. Zur Governance von MaaS: Mobilität erfolgreich steuern. Vortrag auf der Dortmunder Konferenz Raum- und Planungsforschung 2020, Track 6 Mobilität und Verkehr. 17. Februar 2020.

Schwedes, Oliver. 2018. Statt einer Einleitung. In Verkehrspolitik: Eine interdisziplinäre Einführung, Hrsg. Oliver Schwedes. Wiesbaden: Springer.

Schwedes, Oliver. 2017. Verkehr im Kapitalismus. Münster: Dampfboot Verlag.

Schwedes, Oliver. 2014. Fazit: Vom Öffentlichen Verkehr zur Öffentlichen Mobilität. In Öffentliche Mobilität, Hrsg. Oliver Schwedes, 241-251. Wiesbaden: Springer VS.

Schweer, Sebastian. 2014. Skateboarding - Zwischen urbaner Rebellion und neoliberalem Selbstentwurf. Bielefeld: Transcript.

Sheller, Mimi. 2015. Racialized Mobility Transitions in Philadelphia: Connecting Urban Sustianability and Transport Justice. City \& Society 27 (1): 70-91.

Sheller, Mimi und J. Urry. 2000. The City and the Car. International Journal of Urban and Regional Research 24: 737-75.

Spinney, Justin. 2006. Cycling the City: Non-Place and the Sensory Construction of Meaning in a Mobile Practice. In Cycling and Society, Hrsg. Dave Horton, P. Rosen and P. Cox. Hampshire: Ashgate Pubslishing.

SRU - Sachverständigenrat Umwelt. 2005. Umwelt und Straßenverkehr. Hohe Mobilität - Umweltverträglicher Verkehr. Sondergutachten. Berlin: Sachverständigenrat für Umweltfragen.

Steg, Linda. 2005. Car use: lust and must. Instrumental, symbolic and affective motives for car use. Transportation Research A 39: 147-162.

Steg, Lina, C. Vlek, G. Slotegraaf. 2001. Instrumental-reasoned and symbolic-affective motives for using a motor car. Transportation Research Part F 4: 151-169.

Stehlin, John. 2019. Cyclespaces of the unequal city. Bicycle infrastructure and uneven development. Minneapolis, MN: University of Minnessota Press.

Umweltbundesamt. 2019. Veränderungen im Mobilitätsverhalten zur Förderung einer nachhaltigen Mobilität. Abschlussbericht. Dessau-Roßlau: Umweltbundesamt. 
Umweltbundesamt. 2017a. Die Stadt für Morgen: Umweltschonend mobil - lärmarm grün - kompakt - durchmischt. Dessau-Roßlau: Umweltbundesamt.

Umweltbundesamt. 2017b. Umweltbewusstsein in Deutschland. Dessau-Roßlau: Umweltbundesamt.

Urry, John. 2004. The 'System' of Automobility. Theory, Culture \& Society 21 (4-5), 25-39.

Vivanco, Luis. 2013. Reconsidering the Bicycle: An Anthropological Perspective on a New (Old) Thing. London: Routledge.

Wehr, Kevin. 2009. Hermes on Two Wheels: The Sociology of Bicycle Messengers. Lanham et al.: University Press of America.

Williams, Raymond. 1981. Culture. Glasgow: Fontana.

Williams, Raymond. 1976. Keywords: A Vocabulary of Culture and Society. London: Fourth Estate Ltd.

Williams, Raymond. 1961. The Long Revolution. London: Chatto \& Windus.

Open Access Dieses Kapitel wird unter der Creative Commons Namensnennung 4.0 International Lizenz (http://creativecommons.org/licenses/by/4.0/deed.de) veröffentlicht, welche die Nutzung, Vervielfältigung, Bearbeitung, Verbreitung und Wiedergabe in jeglichem Medium und Format erlaubt, sofern Sie den/die ursprünglichen Autor(en) und die Quelle ordnungsgemäß nennen, einen Link zur Creative Commons Lizenz beifügen und angeben, ob Änderungen vorgenommen wurden.

Die in diesem Kapitel enthaltenen Bilder und sonstiges Drittmaterial unterliegen ebenfalls der genannten Creative Commons Lizenz, sofern sich aus der Abbildungslegende nichts anderes ergibt. Sofern das betreffende Material nicht unter der genannten Creative Commons Lizenz steht und die betreffende Handlung nicht nach gesetzlichen Vorschriften erlaubt ist, ist für die oben aufgeführten Weiterverwendungen des Materials die Einwilligung des jeweiligen Rechteinhabers einzuholen.

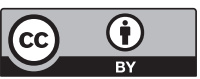

